\title{
1 AutoEM: A Software for Automated Acquisition and Analysis of
}

\section{Nanoparticles}

3 Toni Uusimaeki $\mathrm{i}^{\mathrm{a}}$, Thorsten Wagner ${ }^{\mathrm{b}}$, Hans-Gerd Lipinski ${ }^{\mathrm{b}}$ and Ralf Kaegi ${ }^{\mathrm{a}^{*}}$

4

$5 \quad{ }^{a}$ Eawag, Swiss Federal Institute of Aquatic Science and Technology

$6 \quad{ }^{\mathrm{b}}$ University of Applied Science and Arts Dortmund, Dortmund, Germany

$7 \quad *$ Corresponding author. Tel.: +41 (0)58 76552 73; e-mail: ralf.kaegi@eawag.ch.

8

This document is the accepted manuscript version of the following article:

Uusimaeki, T., Wagner, T., Lipinski, H. G., \& Kaegi, R. (2019). AutoEM: a software for automated acquisition and analysis of nanoparticles. Journal of Nanoparticle Research, 21(6), 122 (11 pp.). https://doi.org/10.1007/s11051-019-4555-9 


\section{1. Abstract}

The era of research on (engineered) nanomaterials (NM) has been thriving for more than a decade and has delivered many beneficial applications, but also raised concerns about potential negative impacts on human health and ecosystems. The precautionary principle, hence, calls for a regulation of certain types of NM, which in consequence requires their unambiguous identification. Most of the currently available definitions of NM rely on an evaluation of the size of the constituent particles and therefore methods have to be developed to measure this parameter. Transmission electron microscopy (TEM) is one of the most promising techniques, as its resolving power well covers the nanosize range. However, limited automation of TEM analyses and possible user bias are major drawbacks of the technique and currently put severe constraints on its broader applications in nanometrology.

Therefore, the goal of this study was to develop a software code, referred to as AutoEM, to automatically acquire TEM images, measure particle sizes and extract the respective particle size distributions (PSD) of (nano)materials. The AutoEM software also incorporates methods for elemental analyses of individual particles using electron energy loss and energy dispersive X-ray spectroscopy (EELS/EDX) allowing the extraction of elementspecific PSDs. Additionally automated acquisition of energy filtered images (EFTEM) is implemented in the AutoEM software, which can be used e.g. to derive thickness maps and, thus, to evaluate the thickness of individual (plate-like) particles. 


\section{Introduction}

Technological advancements increasingly stimulate research and development of engineered nanomaterials (NM) to capitalize unique and novel properties occurring at the confined nanoscale dimensions. This has resulted in a plethora of NM which today can be found in many technological applications and consumer products (Caballero-Guzman and Nowack 2016; Kanchi et al. 2018). Although NM are designed to increase profitability and sustainability, unknown impacts on human health and ecosystems raised concerns from the very beginning about potential risks associated with the increased use of NM (Roco and Bainbridge 2005; Wiesner et al. 2006; Nowack and Bucheli 2007; Keller et al. 2013; Gottschalk et al. 2013; Giese et al. 2018). In a regulatory framework this means that NM have to be clearly defined and analytical methods have to be available to unambiguously identify NM based on the respective definition. For example, the recommendation of a definition of a NM released by the European Commission (EC) - for convenience, later referred to as EC definition - states that a particulate material is a NM if the median $\left(\mathrm{X}_{50}\right)$ of the number based particle size distribution (PSD) is between 1- $100 \mathrm{~nm}$ (European Commission 2011). The implementation of such a definition, however, is only meaningful, if methods that cover the respective size range are available.

Multitudes of methods to obtain a PSD of particulate material have been reviewed and described in the scientific literature, with each method having its limitations and area of application (Hassellöv et al. 2008; Hassellöv and Kaegi 2009; Farre et al. 2009; von der Kammer et al. 2011; Laborda et al. 2016a; Babick et al. 2016). However, only a few methods deliver number based PSDs (e.g. particle tracking analyses (Hole et al. 2013), single particle inductively coupled mass spectrometry (sp-ICPMS (Pace et al. 2012; Laborda et al. 2016b)) and all of these methods do not cover the whole nanosize range as required by the EC definition. Furthermore, the EC definition refers to the minimal external dimension of a particle as the particle size. Advanced light scattering techniques based on single particle extinction and scattering have recently demonstrated the possibility to assess various shape descriptors of anisotropic gold nanoparticles (NP) (Potenza et al. 2017). However, assessing different shape descriptors such as the length and width of rods or the thickness of plate-like particles is very challenging or even impossible for currently established particle sizing techniques such as dynamic light scattering or spICPMS, as the particle size derived from these techniques results from a conversion of recorded signal intensities into equivalent spherical particles. As a result, very contrasting PSDs with $\mathrm{X}_{50}$ values ranging from a few tens to a few hundreds of nanometers were reported for plate-like particles measured with different measurement techniques (Babick et al. 2016). These, to some extent disillusioning results, clearly reveal the lack of methods capable of accessing minimal external diameters in three dimensions. 
TEM allows a visualization of the lateral extent of individual particles (critical for rod-like particles) and with its superior resolving power, TEM covers the entire nanosize range $(1-100 \mathrm{~nm})$ and, depending on the instrumental settings, is also capable of recording particles up to a few microns. Recorded TEM images have to be postprocessed (e.g. segmentation to separate particles from the background and to resolve overlapping particles) to obtain a number based PSD. Classical TEM bright field (BF) images are essentially two dimensional (2D) projections of the particles based on mass-thickness and /or diffraction contrast, and a multitude of diameters such as minimal lateral extension or equivalent circular diameter (ECD) can be extracted from the recorded images (ISO/TC 24/SC 4). This reflects one of the biggest advantages of the TEM technique in combination with image analyses tools. For rod-like particles, for example, the ECD may not be a very useful parameter and the length and width of the rods are probably more relevant shape descriptors. Furthermore, for the EC definition, the minimum external dimensions of the particles have to be used to construct the particle size distribution. Several image analysis programs and algorithms (Cervera Gontard et al. 2011; Mondini et al. 2012; Park et al. 2013) and numerous plugins to ImageJ (Schneider et al. 2012) are available for particle segmentation. The TEM is, therefore, often considered as one of the most reliable measurement techniques in the field of nanometrology (Dudkiewicz et al. 2015), but the inability to access the third dimension of plate-like particles using conventional TEM is a limitation of the method. Electron tomography can be applied to recover the morphology of individual NPs in all three dimensions, but this method is still very time consuming and thus results in an extremely low particle (and consequently sample) throughput (Thomas et al. 2013; Moldovan et al. 2015). However, more advanced TEM techniques, such as EFTEM, offer alternative ways to assess the thickness of nanostructures (Potapov 2014) and overcome the limitations posed by conventional TEM analyses and may thus fill an important gap regarding the unambiguous classification of (plate-like) NMs. Despite all the striking advantages of TEM, the technique is still labor intensive and has a lower throughput as compared to other currently available methods. To overcome these limitations, we developed a software environment referred to as AutoEM which i) allows the automatic recoding and processing of TEM and scanning TEM (STEM) images, ii) implements elemental analyses of individual particles for the extraction of elementally resolved PSDs and iii) includes the possibility to automatically record EFTEM images to derive thickness maps and thereby to access the third dimension of plate-like particles. As a proof on concept, examples covering imaging, both TEM-BF and STEM dark field (DF), EDX spectrum imaging (SI) and EFTEM thickness mapping are provided and discussed in this work.

\section{Materials and Methods}




\subsection{Preparation of TEM grids suitable for the autoEM analyses}

To demonstrate the potential of the AutoEM software, TEM grids (carbon coated $\mathrm{Cu}$ grids, EM solutions, UK) were prepared from i) a colloidal silica suspension (particle diameter $=27.8 \mathrm{~nm}$, ERM FD 304) ii) monomodal

silver (Ag, $20 \mathrm{mg} \mathrm{L}^{-1}$, diameter $40 \mathrm{~nm}$, stabilized in $0.2 \mathrm{mM}$ citrate, Nanocomposix) and monomodal gold (Au, $50 \mathrm{mg} \mathrm{L} \mathrm{L}^{-1}, 40 \mathrm{~nm}$, stabilized in $0.2 \mathrm{mM}$ citrate, Nanocomposix) suspensions and iii) a nanoclay (illite) suspension (15.88 g Illite $\mathrm{L}^{-1}$, described in Wick et al. (2018)).

All four particle types were expected to carry an negative surface charge under the given conditions, and thus, TEM grids were functionalized using poly-l-lysine (PLL) to positively charge the TEM grids (Prasad et al. 2015). All TEM grids were prepared by directly centrifuging particle suspensions on TEM grids (Mavrocordatos and Perret 1995) after appropriate dilution of the respective stock suspensions. The dilutions for the colloidal silica (1:2) and the illite (1:50) suspensions were obtained using doubly deionized (DDI) water. The dilutions of the $\mathrm{Au}(1: 50)$ and the $\mathrm{Ag}(1: 40)$ suspensions were performed in 2 mmol sodium citrate solutions. All suspensions were sonicated for 1 minute using a vial tweeter (Vial Tweeter, Hielscher Ultrasonics GmbH, Germany). For the centrifugation procedure, aluminum cones were covered with a thin film of plastic (PARAFILM, Sigma-Aldrich) and TEM grids were gently pressed onto this film. The aluminum cones were inserted into standard Eppendorf centrifugation tubes $(2 \mathrm{~mL})$ and filled with $1 \mathrm{~mL}$ of the working suspensions. A video explaining the procedure in detail is available in the internet (ParticleLab). The centrifugation conditions were kept constant for all TEM grids $(14,000 \mathrm{x}$ g, $1 \mathrm{~h})$ and were sufficient to bring all the particles to the surface of the aluminum cone and thus to deposit the particles onto the TEM grid.

\subsection{System Requirements}

The AutoEM software is developed within the Gatan Digital Micrograph ${ }^{\mathrm{TM}}$ (DM) environment (Mitchell and Schaffer 2005) and allows a fully automated acquisition of (S)TEM images with an online post-processing of recorded images and a 'live' display of the extracted PSD. For this purpose, the AutoEM software is coupled with the NanoDefine ParticleSizer (ND-PS) (Wagner 2016), written in ImageJ / Fiji open source platform (Schindelin et al. 2012; Schneider et al. 2012). The ND-PS is a segmentation software specially developed for separating overlapping (agglomerated or aggregated) particles. A numerically optimized and multithreaded nonlocal means filter is used to smooth the images (Wagner and Behnel 2015) before the segmentation process. The settings of the ND-PS allows the user to choose between the following four modes: the default mode for nonoverlapping particles, normal and irregular watershed mode for overlapping particles and ellipse fitting mode for 
overlapping, ellipsoidal particles. Additionally a single particle mode is available, which uses a user-defined convexity value as a threshold to reject agglomerates and, thus, to only include primary particles.

The AutoEM software uses DM to control the TEM, hence DM has to be present at the microscope with the related microscope and camera commands installed. Additionally, STEM mode requires Digiscan hardware to control the electron beam, and STEM EDX/EELS requires the spectral imaging plugin installed in DM. EFTEM mode requires Gatan imaging filter (GIF) and the related control commands. The AutoEM software has been successfully tested with all three versions of DM (PC only), with three different FEI microscopes (2x TF20, T12) and with three different JEOL microscopes (2100F, 2800 and 3200FSC).

\subsection{Software Development}

With the basic version of the AutoEM software, one can acquire and evaluate images serially both in TEM BF or STEM DF mode, with a live update of the extracted PSD through an interface with the ND-PS. However, any other contrast mechanisms e.g. TEM DF, phase-contrast or STEM BF and STEM high-angle annular dark-field (HAADF) can also be used for image formation in combination with the AutoEM software. A more advanced version of the AutoEM software includes options for i) EFTEM (extraction of thickness maps or core-loss imaging for elemental information), ii) STEM EDX (elemental information), and iii) STEM EELS (elemental information). Within the work flow of the data acquisition and analysis of the autoEM software, there are several empty functions, allowing an easy extension of the software capabilities by the end user via scripting.

The basic version of the AutoEM software user interface (UI) consists of the following three tabs: camera, main and settings. The camera tab is used for setting the imaging and auto-focusing conditions; an auto-exposure option is also provided. In the main tab, the most common measurands e.g. feret min or equivalent circular diameter (ECD) can be selected for creating the PSD. Feret min refers to the minimum distance between two parallel lines constraining the particle, and the ECD is the diameter of a circle with an equivalent area as the projection of the particle of interest. Several (larger) fields of interest, each containing multiple images can be defined. Furthermore, the number of images used to create the image montage at high magnification can be defined in the main tab. The necessary calibrations in the settings tab include the magnification list and stage calibration. The former reads the pixel sizes of all magnifications and has to be performed only once. The latter relates the stage to the camera coordinates and has to be done for each magnification and camera separately. If a large montage image is required with a precise overlap between two subsequently acquired images, image shift will be used. This requires image shift, beam shift and image shift induced beam shift calibration to be 
performed (TEM mode). Additionally auto-exposure and auto-focusing routines require calibration steps, which are described in detail in the installation manual of the autoEM software. A default delay time after stage movements of $6 \mathrm{~s}$ is included in the autoEM software to allow the specimen to stabilize and, thus, to avoid degrading image qualities related to specimen drift. The delay time can be adjusted by the user. Furthermore, stage drift corrections and autofocusing routines are performed after recording the EDX spectra of a given number of particles, which can be defined by the user. The accumulated beam shift resulting from these corrections is then included in following stage movements.

The EDX option adds another tab to the UI with an elemental table. From there the user can choose the elements and associated peaks for the identification of individual particles. A spectrum image will be recorded for each particle (EDX and/or EELS) at a user-defined resolution. Spectrum imaging (SI) allows recording multiple EDX spectra of one particle by sequentially scanning the electron beam over the particle of interest and recording EDX spectra in a point-by-point manner. SI of individual particles therefore provides more reliable information of the elemental composition of individual particles, as the whole particle is scanned by the electron beam, compared to a 'point' analysis where the elemental information only originates from a very limited volume of the particle. Furthermore, spatially resolved elemental analyses as provided by SI may also allow detecting uneven distributions of certain elements within individual particles, such as core-shell structures. For the detection of individual elements present in a particle of interest all the EDX spectra in the SI are integrated and smoothed (Savitzky and Golay 1964) to produce one EDX spectrum for every individual particle. The detection of individual peaks at element specific energies is based on a simple threshold criterion. For STEM EELS SI, only automatic acquisition is provided and further analysis and interpretation of the data requires offline processing by the user.

EFTEM incorporates a large family of methods for analysis. The user can add any number of edges with specific slit widths and exposure times. This provides the basis for e.g. thickness mapping, zero-loss filtering, core loss and pre-carbon imaging, jump ratio images and the three windows - method (Reimer 1995). The AutoEM software serially records a suite of filtered (zero-loss) and unfiltered images, required to calculate thickness maps. The log-ratio of the filtered and unfiltered images is referred to as the relative thickness map, which can be converted into absolute thickness map by multiplication with the electron mean free path lengths for inelastic scattering $(\lambda)$ in the respective material. For standard materials, $\lambda$ values are tabulated or can be found in respective publications, or alternatively it can be calculated based on theoretical grounds (Malis et al. 1988; Plitzko and Mayer 1999; Lee et al. 2002; Iakoubovskii et al. 2008; Meltzman et al. 2009; Craven et al. 2016). 
For this work, $\lambda$ value of $127.1 \mathrm{~nm}$ for illite was determined using the mean free path estimator script provided by (Mitchell).

The intensity variations in the (relative) thickness map images correspond directly to the roughness of the surface topology of the 3D particle in the $\mathrm{z}$ direction (direction of the electron beam). The acquired images were corrected by removing X-ray spikes (corresponding to pixels of very high signal intensities). If the pixel values exceed a user specified threshold, then the respective pixel value was replaced by the mean value of its next neighbors. Furthermore, the images were processed with a low pass filter with Gaussian smoothing to reduce the noise in the relative thickness maps before calculating the log-ratio of zero-loss and unfiltered images. The thickness maps were multiplied with the segmented images that were derived by processing the zero-loss image using the ND-PS, resulting in a corrected thickness map with clearly defined particles above a zero background. This corrected thickness map was multiplied with the calculated $\lambda$ value to convert the relative particle thickness into absolute thickness values. The mean log-ratio value of the carbon film was calculated by using the segmented images and excluding areas where particles were identified. It was assumed that the thickness of the carbon film remained constant throughout the TEM grid, and therefore, the height obtained for the particles was corrected for the height obtained for the carbon film underlying the deposited particles. The intensity values of the corrected thickness map were then extruded into $\mathrm{z}$ dimension, and displayed using the same scaling as in $\mathrm{x}$ and y dimension. The coordinates were adjusted evenly into both directions of the z-axis, assuming that particles follow a mirror symmetry with respect to the z zero-plane. This assumption holds true e.g. for spherical, ellipsoidal and cubic particles, but fails for particles irregular in the $\mathrm{z}$ dimension. However, for plate-like particles as investigated in this study, we expect that the symmetry assumption is fulfilled. Due to the symmetry constraints, we call this reconstruction quasi 3D (q3D). Both 2D and q3D segmented images were processed using the Avizo software code (Thermo Fisher Scientific Inc.). A DM script UI is included in the download folder to reconstruct q3D particle morphologies from acquired elastic and unfiltered images. The AutoEM software is freely downloadable from the following location accompanied with a manual providing more information about the software, its installations, required calibration steps and additional options:

https://doi.org/10.5281/zenodo.2598435

Examples of customizable scripts have been included in the download folder, which shows how to perform userdefined tasks at various locations in the workflow e.g. how to place the electron beam in STEM mode to the middle of the particles and record a convergent beam electron diffraction (CBED) image using the CCD camera. 
designed for single particle imaging - the AutoEM software is specifically designed for NP detection and analyses, including for example elemental analyses of individual particles, the extraction of (element specific) PSD and the determination of the mean thickness of plate-like (nano)particles.

\section{Results and Discussion}

\subsection{Conventional TEM BF and STEM DF Mode}

To demonstrate the capabilities of the AutoEM software to record images suitable for extracting a PSD, different TEMs were operated in both, basic TEM and STEM mode. Colloidal silica NPs (ERM-FD304) with an indicative mean ECD of $27.8 \mathrm{~nm}$ (Franks et al. 2012) were deposited on carbon coated TEM grids by centrifugation and served as examples for this case study.

For TEM BF imaging, a $60 \mathrm{kx}$ magnification was used, resulting in a pixel size of $1.48 \mathrm{~nm}$ and a field of view (FOV) of $3 \mu \mathrm{m}$. A particle of a diameter of $20 \mathrm{~nm}$ thus consists of $\sim 570$ pixels, well above the limit of 100 pixels per particles suggested by other studies (Schöpe et al. 2007; Pyrz and Buttrey 2008; Merkus 2009) for detecting and sizing particles based on electron microscopy analyses. The magnification calibration was performed using an Agar scientific cross grating sample with the same defocus applied as in the acquisition process. A montage image, consisting of as 5 x 10 images was recorded with the AutoEM software (Figure 1a), resulting in a total FOV of $15 \times 30 \mu \mathrm{m}$. The offline segmentation of the images was performed using the ND-PS (default settings) and in total 6400 particles were detected. The extracted size distribution revealed a mean ECD value of $29.4 \mathrm{~nm} \pm 6.1 \mathrm{~nm}(1 \sigma)$, in excellent agreement with the indicated value of $27.8 \mathrm{~nm}( \pm 1.5 \mathrm{~nm}$ corresponding to a confidence level of 95\%) for the colloidal silica derived from EM measurements.

For STEM HAADF imaging, a magnification $200 \mathrm{kx}$ was chosen, resulting in a pixel size of $1.15 \mathrm{~nm}$ and a FOV of $1.17 \mu \mathrm{m}$. A montage image consisting of $4 \times 10$ images was recorded (Figure 1b), corresponding to a total FOV of $4.7 \times 11.7 \mu \mathrm{m}$. The offline segmentation was again performed using the ND-PS with default settings and in total 1152 particles were detected. The extracted mean ECD of $27.7 \mathrm{~nm} \pm 3.6 \mathrm{~nm}(1 \sigma)$ excellently matched the indicated value of $27.8 \mathrm{~nm}$. These results demonstrate that the modal diameter of the investigated colloidal silica reference material obtained from automatically recorded and evaluated TEM images using the AutoEM software operated in both TEM and STEM modes, is well within the uncertainty limits of the indicated value of the respective reference material. This sets the basis for further exploring the capabilities of the AutoEM software, namely i) to distinguish between different particles types having the same size but different elemental 


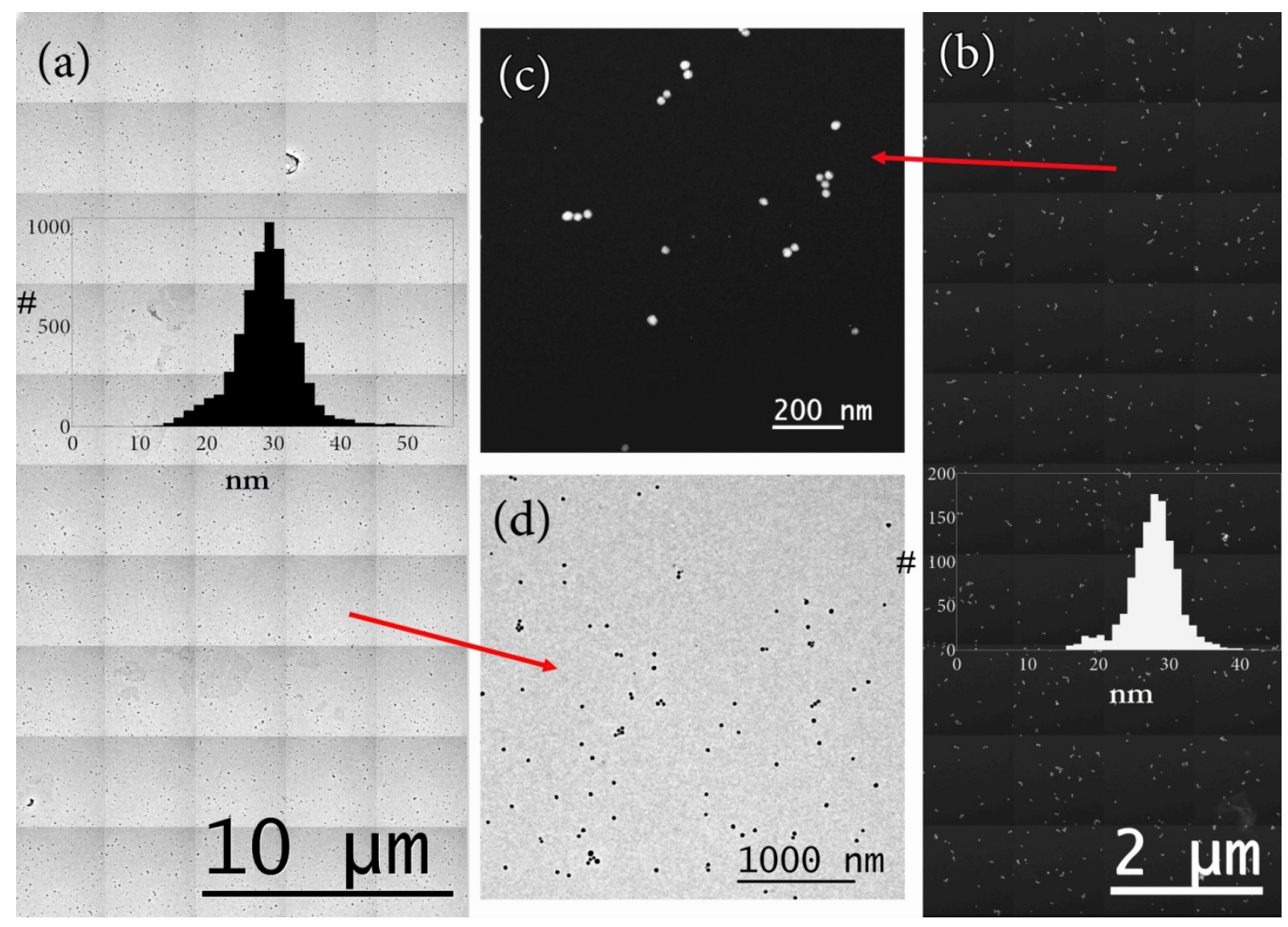

Figure 1. (a) TEM and (b) STEM montage images with automatically extracted particle size distributions using the ND-PS operated with default settings. (c) and (d) represent individually acquired images from (b) and (a) respectively.

\subsection{Elemental Specific PSDs Derived from EDX Measurements}

To assess the capabilities of the AutoEM software to distinguish between particles of different elemental compositions, a mix of Au- and Ag-NPs, both having a diameter of $40 \mathrm{~nm}$, was centrifuged onto C-coated TEM grids. As the focus of this experiment was to demonstrate the feasibility to record EDX spectra of individual NP which may be even touching each other, Au and Ag particles, both showing strong contrast in STEM-HAADF images, were selected. For the analyses of the elemental composition of individual particles, the AutoEM software was operated in STEM mode. This allowed i) an automatic acquisition of images in a sequential 
manner, ii) conducting an online segmentation using the ND-PS operated in default settings and ii) recording an EDX spectrum of every identified particle separately.

A montage image consisting of 2 x 2 images (TEM grid with the Au-, Ag-NP mix) was acquired on two different areas, including 10 seconds of SI acquisition for every particle. The stage drift was automatically corrected after every 10 particles using sub-pixel cross-correlation and beam shift. Automatic focusing was performed every 40 particles. An individual HAADF image is given in Figure 2a with an example of a SI of a Au- and a Ag-NP given in Figures $2 \mathrm{~b}$ and $2 \mathrm{c}$. The integrated spectrum from the respective Au and Ag-NP is provided in Figures $2 \mathrm{~d}$ and 2e, respectively. Based on the integrated EDX spectra, the particles were classified as either Au or Ag-NPs and the respective PSD of both particle types are given in Figures $2 \mathrm{f}$ and $2 \mathrm{~g}$. In total $748 \mathrm{Au}-$ and $748 \mathrm{Ag}-\mathrm{NPs}$ were detected with a median ECD of $39.3 \mathrm{~nm} \pm 4.6 \mathrm{~nm}(1 \sigma)$ for the Au-NPs and $37.7 \mathrm{~nm} \pm 4.7 \mathrm{~nm}(1 \sigma)$ for the Ag-NPs. This is very comparable to the size information provided by the manufacturer which were $40 \pm 4 \mathrm{~nm}$ (mean value) for both particle types. In addition to the 1496 spectra which were assigned to either Au- or AgNPs, 11 spectra remained unassigned, because of lack of signal intensities at the beginning of the EDX recording, which most likely was related to initial communication errors between the EDX detector and DM.

The entire measurements, including the elemental analyses were conducted on a JEOL JEM-2100F equipped with a Schottky-type field emission gun and lasted for $9 \mathrm{~h}$ in total. However, new EDX detectors with increased collection angles, the installation of multiple EDX detectors and novel high brightness emission guns will result in higher signals intensities emitted from the sample and in a more efficient collection of the X-rays. Therefore, the acquisition time may be reduced by at least a factor of $10-15$ resulting in an analyses time of less than $1 \mathrm{~h}$. Therefore, in addition to the time required for the analyses of the samples in the electron microscope, the time used for sample preparation will become increasingly important regarding sample throughput and / or costs per 

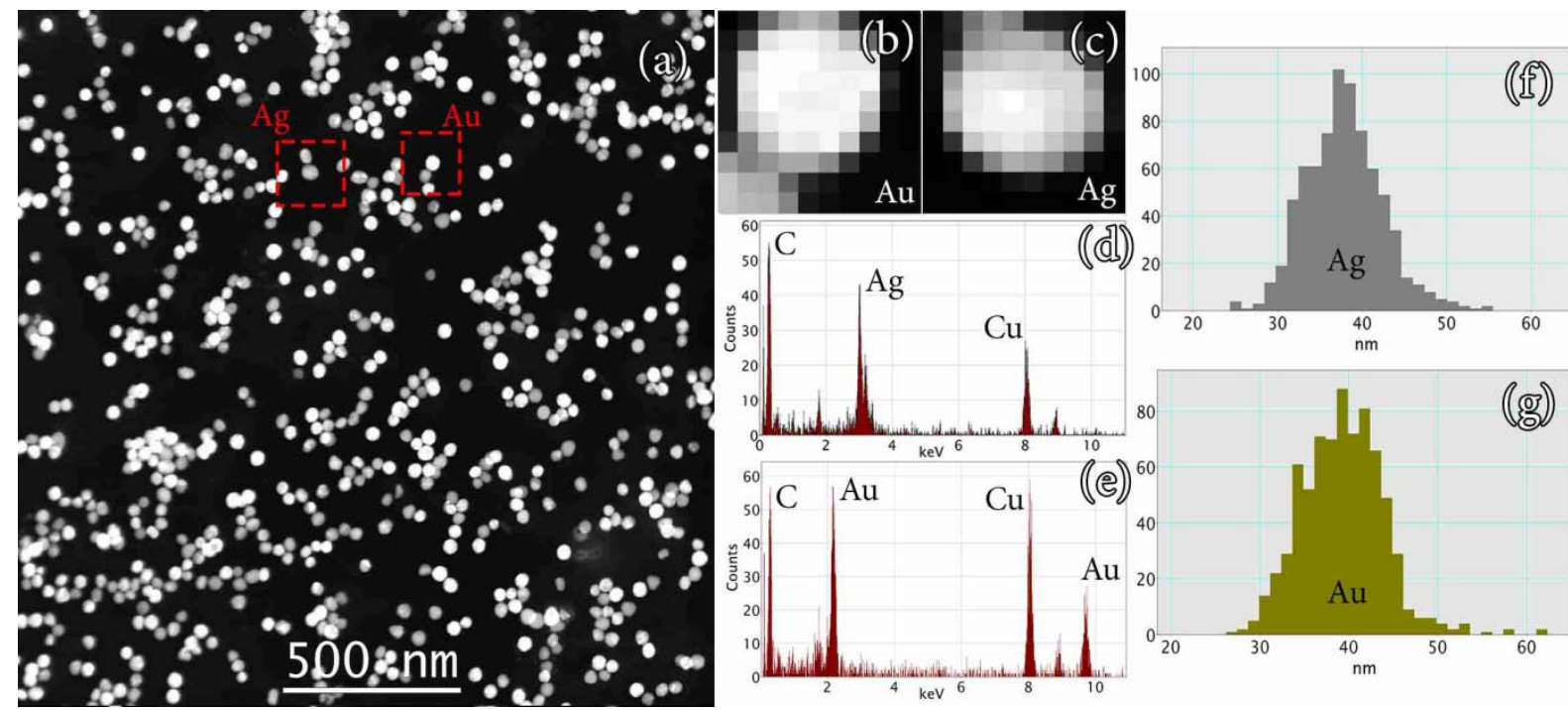

Figure 2. (a) STEM-HAADF image of the Au - Ag-NPs mix deposited on a TEM grid. (b,c) Spectrum images acquired from (a). (d,e) Integrated EDX spectra used for particle identification. (f,g). Elementally resolved PSDs (based on the ECD) of Ag- and Au-NPs, respectively.

\subsection{Thickness Mapping - Accessing the Third Dimension of Plate-like Particles}

To demonstrate the capabilities of the AutoEM software to also access the thickness of plate-like particles, we investigated a TEM grid prepared with clay particles (illite) and acquired a series (10 x 16) of zero-loss filtered and unfiltered images. A montage image of the 160 zero-loss filtered images is provided in Figure 3a. The pixel size corresponded to $1.16 \mathrm{~nm}$, resulting in a total FOV of $23.7 \mu \mathrm{m} \times 38 \mu \mathrm{m}$. Zero-loss images were recorded with an acceleration voltage of $200 \mathrm{kV}$, using a slit width of $20 \mathrm{eV}$ and a semi-collection angle of $32.3 \mathrm{mrad}$. Pairs of unfiltered (e.g. Figure 3b) and the zero-loss filtered (elastic) (e.g. Figure 3c) images were cross-correlated to correct for a possible stage shift.

In total approximately $18^{\prime} 000$ particles were detected and the PSD extracted from these images resulted in a (feret min) median diameter of $66.5 \pm 56.9 \mathrm{~nm}(1 \sigma) \mathrm{nm}$ corresponding to the minimum lateral extent (2D) of the illite platelets. The considerable standard deviation obtained for the lateral extent of the illite platelets reflects the polydispersity of this natural material and highlights an additional challenge associated with the EC definition. Although the mode of a PSD may be larger than $100 \mathrm{~nm}$, and thus the respective material would not be classified as a NM, depending on the polydispersity and the modal value of the PSD, variable fractions of particles will be 
every image was calculated (Figure 3f, g). The ratio of the thickness (t) to the mean free path length of the electrons $(\mathrm{t} / \lambda)$ was always $<1$, and as such plural scattering effects can be neglected. An analysis of the q3D reconstruction resulted in a median thickness of the particles of $12.4 \mathrm{~nm} \pm 8.9 \mathrm{~nm}(1 \sigma)$, which is more than a factor of 5 smaller compared to the $2 \mathrm{D}$ result. Both, thickness and diffraction will contribute to the image contrast in the $\mathrm{BF}$ images. The influence of diffraction contrast to thickness mapping is discussed e.g. by (Mitchell 2006). Depending on the crystallinity and density of the material, the accuracy on the thickness obtained through the evaluation of $\lambda$ may vary between $5 \%$ and $20 \%$ and it is recommended to use collection angles larger than $25 \mathrm{mrad}$ at $200 \mathrm{kV}$ (Nakafuji et al. 2001; Lee et al. 2002). In the context of the EC definition of a nanomaterial, an overestimation of the thickness by $20 \%$ would transfer into a size (thickness) limit of $\sim 120$ $\mathrm{nm}$ instead of $100 \mathrm{~nm}$ for plate like particles. However, with a mean thickness of $10-15 \mathrm{~nm}$, the clay particles investigated in this study are clearly classified as a nanomaterial according to the EC definition, also if an uncertainly in the order of $20 \%$ is included.

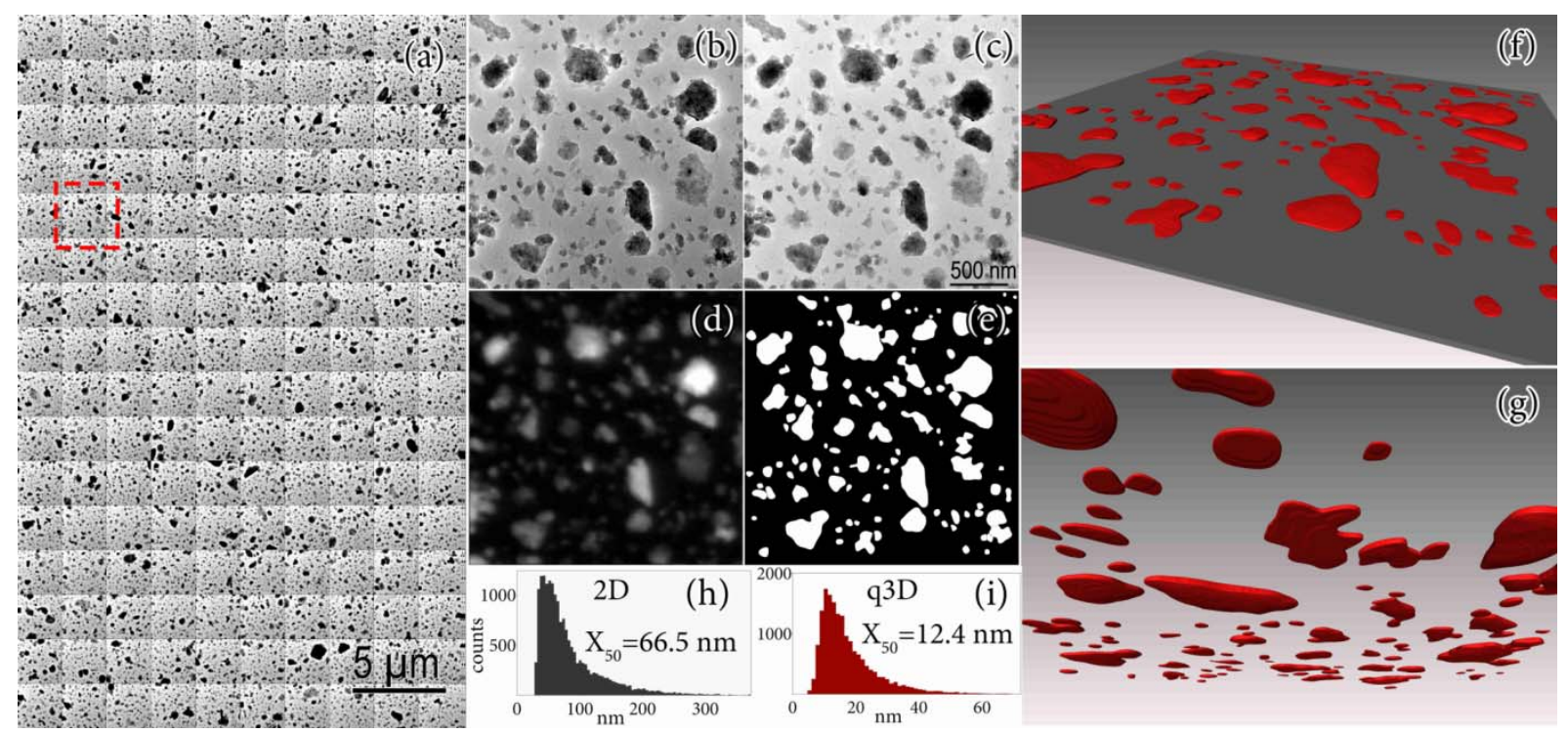

Figure 3. (a) A zero-loss montage image of illite NPs. Selected unfiltered (b) and zero-loss filtered (c) images of the illite sample. The red square in (a) represents the selected image. (d) Calculated thickness map of the image marked with the red square in (a) and the resulting segmentation (e) using the ND-PS with default mode settings. (h,i) 2D and q3D particle size distribution respectively, derived by processing all images shown in (a). (f,g) q3D reconstruction of selected particles from above with the TEM grid (carbon film included) and (g) from below the TEM grid without the carbon film.

\section{Conclusions}


We have developed a software (AutoEM) for automated acquisition and analyses of a series of electron microscopy images (TEM and STEM). The AutoEM software is interfaced with an image analyses tool (ND-PS) allowing an 'on-line' extraction and display of the number based PSD of particles deposited on TEM grids, even during ongoing TEM acquisition. The AutoEM software allows the microscopes to be operated in both TEM and STEM mode and almost identical results were obtained from both operation modes when colloidal silica particles (ERM-FD304) were investigated. The median of the extracted PSD was always within the uncertainty bounds of the indicated reference values of the ERM material. The flexible architecture of the AutoEM software allows an easy extension of the basic TEM and STEM operation modes for fully exploiting the potential of TEM techniques. By the combination with EDX analyses, for example, Au- and Ag-NPs of the same size were successfully separated into different particle categories. By employing more advanced TEM techniques such as EFTEM, plate-like particles (illite in this specific case) were characterized in $\mathrm{q} 3 \mathrm{D}$ and the minimal external diameter corresponding to the plate thickness was extracted. Although in this specific example, the illite material would be classified as NM based on both the $2 \mathrm{D}$ and the $\mathrm{q} 3 \mathrm{D}$ analyses, it may be well conceivable that other plate-like materials may be classified as NMs based on a $\mathrm{q} 3 \mathrm{D}$ evaluation, but would not be considered as NMs when only characterized based on a $2 \mathrm{D}$ evaluation. As the EC definition of a NM specifically states that the PSDs should be established based on the minimal external dimension of the particles of interest, assessing the third dimension of plate-like particles in mandatory, which can be achieved by automated EFTEM imaging as offered by the AutoTEM software in combination with q3D analysis of individual (nano)particles.

The presented AutoEM software offers a very comprehensive solution for the characterization of NMs according to the EC definition, also for very challenging particles types with plate-like morphologies. The capabilities of this software can easily be extended by the end user allowing for example the classification of particles according to their crystallographic structures through diffraction mapping. The limitations of the software are directly coupled to the imaging modes of the (S)TEM itself, and particles in the lower nm size range can be investigated as long as there is sufficient contrast to distinguish the particles from the background. The achievable detection limits will therefore be dependent on the elemental composition of the particles of interest and on the signal used for image formation. In addition, sample contamination in the electron microscopy during analyses may additionally constrain the achievable detection limits. Thus, the practical detection limits will have to be evaluated case-by-case. The increased degree of automation offered by the autoEM software in combinations with reduced analyses times due to increased signal intensities obtained with newer (S)TEMs likely shift the bottleneck for NP analyses from the actual time required on the microscope towards the time needed for the preparation of TEM samples suitable for automated analyses. 


\section{Acknowledgements}

This work was accomplished within the NanoDefine project - and has been made possible from the funding of European Community's Seventh Framework Programme (FP7/2007-2013) under Grant Agreement $n^{\circ} 604347$ and Grant Agreement 312483 - ESTEEM2 (Integrated Infrastructure Initiative I3). Additionally the authors would like to thank ScopeM (ETH), Felmi-ZFE (TU Graz), Center for Microscopy and Image Analysis (UZH), Department of Materials and Environmental Chemistry (Stockholm University), Nanomicroscopy Center (Aalto University) and Electronmicroscopy Unit (University of Helsinki) for collaboration and technical support. A special appreciation belongs to Dr. Bernhard Schaffer (Gatan) for helpful discussions and support. Andreas Voegelin is acknowledged for providing illite suspensions.

\section{Conflict of Interest:}

The authors declare that they have no conflict of interest.

\section{References}

Babick F, Mielke J, Wohlleben W, et al (2016) How reliably can a material be classified as a nanomaterial? Available particle-sizing techniques at work. J Nanoparticle Res 18:158. doi: 10.1007/s11051-0163461-7

Caballero-Guzman A, Nowack B (2016) A critical review of engineered nanomaterial release data: Are current data useful for material flow modeling? Environ Pollut 213:502-517. doi: 10.1016/j.envpol.2016.02.028

Cervera Gontard L, Ozkaya D, Dunin-Borkowski RE (2011) A simple algorithm for measuring particle size distributions on an uneven background from TEM images. Ultramicroscopy 111:101-106. doi: 10.1016/j.ultramic.2010.10.011

Craven AJ, Bobynko J, Sala B, MacLaren I (2016) Accurate measurement of absolute experimental inelastic mean free paths and EELS differential cross-sections. Ultramicroscopy 170:113-127. doi: 10.1016/j.ultramic.2016.08.012

Dudkiewicz A, Boxall ABA, Chaudhry Q, et al (2015) Uncertainties of size measurements in electron microscopy characterization of nanomaterials in foods. Food Chem 176:472-479. doi: 10.1016/j.foodchem.2014.12.071

European Commission (2011) Commission Recommendation of 18 October 2011 on the definition of nanomaterial. Off J Eur Union L 275:38-40

Farre M, Gajda-Schrantz K, Kantiani L, Barcelo D (2009) Ecotoxicity and analysis of nanomaterials in the aquatic environment. Anal Bioanal Chem 393:81-95. doi: 10.1007/s00216-008-2458-1 
Franks K, Braun A, Charoud-Got J, et al (2012) Certification of the equivalent spherical diameters of silica nanoparticles in aqueous solution - Certified Reference Material ERM $®-F D 304$ - EU Science Hub European Commission. In: EU Sci. Hub. https:/ec.europa.eu/jrc/en/publication/eur-scientific-andtechnical-research-reports/certification-equivalent-spherical-diameters-silica-nanoparticles-aqueoussolution-certified. Accessed 16 Nov 2018

Giese B, Klaessig F, Park B, et al (2018) Risks, Release and Concentrations of Engineered Nanomaterial in the Environment. Sci Rep 8:1565. doi: 10.1038/s41598-018-19275-4

Gottschalk F, Sun T, Nowack B (2013) Environmental concentrations of engineered nanomaterials: Review of modeling and analytical studies. Environ Pollut 181:287-300. doi: 10.1016/j.envpol.2013.06.003

Hassellöv M, Kaegi R (2009) Analysis and Characterization of Manufactured Nanoparticles in Aquatic Environments. In: Lead JR, Smith E (eds) Environmental and Human Health Impacts of Nanotechnology. John Wiley \& Sons, Ltd, pp 211-266

Hassellöv M, Readman JW, Ranville JF, Tiede K (2008) Nanoparticle analysis and characterization methodologies in environmental risk assessment of engineered nanoparticles. Ecotoxicology 17:344361. doi: 10.1007/s10646-008-0225-x

Hole P, Sillence K, Hannell C, et al (2013) Interlaboratory comparison of size measurements on nanoparticles using nanoparticle tracking analysis (NTA). J Nanoparticle Res 15:UNSP 2101. doi: 10.1007/s11051013-2101-8

Iakoubovskii K, Mitsuishi K, Nakayama Y, Furuya K (2008) Mean free path of inelastic electron scattering in elemental solids and oxides using transmission electron microscopy: Atomic number dependent oscillatory behavior. Phys Rev B 77:104102. doi: 10.1103/PhysRevB.77.104102

ISO/TC 24/SC 4 ISO 13322-1:2014 - Particle size analysis -- Image analysis methods -- Part 1: Static image analysis methods. https://www.iso.org/standard/51257.html. Accessed 22 Oct 2018

Kanchi S, Ahmed S, Sabela M, Hussain C (2018) Nanomaterials : biomedical, environmental, and engineering applications / editors: Suvardhan Kanchi [and 3 more]. John Wiley \& Sons, Hoboken, NJ

Keller AA, McFerran S, Lazareva A, Suh S (2013) Global life cycle releases of engineered nanomaterials. J Nanoparticle Res 15:1692

Laborda F, Bolea E, Cepriá G, et al (2016a) Detection, characterization and quantification of inorganic engineered nanomaterials: A review of techniques and methodological approaches for the analysis of complex samples. Anal Chim Acta 904:10-32. doi: 10.1016/j.aca.2015.11.008

Laborda F, Bolea E, Jimenez-Lamana J (2016b) Single particle inductively coupled plasma mass spectrometry for the analysis of inorganic engineered nanoparticles in environmental samples. Trends Environ Anal Chem 9:15-23. doi: 10.1016/j.teac.2016.02.001

Lee C-W, Ikematsu Y, Shindo D (2002) Measurement of mean free paths for inelastic electron scattering of Si and SiO2. J Electron Microsc (Tokyo) 51:143-148. doi: 10.1093/jmicro/51.3.143

Malis T, Cheng SC, Egerton RF (1988) EELS log-ratio technique for specimen-thickness measurement in the TEM. J Electron Microsc Tech 8:193-200. doi: 10.1002/jemt.1060080206

Mavrocordatos D, Perret D (1995) Non-Artifacted Specimen Preparation for Transmission Electron-Microscopy. Commun Soil Sci Plant Anal 26:2593-2602. doi: 10.1080/00103629509369470

Meltzman H, Kauffmann Y, Thangadurai P, et al (2009) An experimental method for calibration of the plasmon mean free path. J Microsc 236:165-173. doi: 10.1111/j.1365-2818.2009.03214.x

Merkus H (2009) Particle Size Measurements - Fundamentals, Practice, Quality. https://www.springer.com/de/book/9781402090158. Accessed 21 Mar 2019 
Mitchell DRG Mean Free Path Estimator. http://www.dmscripting.com/meanfreepathestimator.html. Accessed 21 Mar 2019

Mitchell DRG (2006) Determination of mean free path for energy loss and surface oxide film thickness using convergent beam electron diffraction and thickness mapping: a case study using Si and P91 steel. J Microsc 224:187-196. doi: 10.1111/j.1365-2818.2006.01690.x

Mitchell DRG, Schaffer B (2005) Scripting-customised microscopy tools for Digital Micrograph ${ }^{\mathrm{TM}}$. Ultramicroscopy 103:319-332. doi: 10.1016/j.ultramic.2005.02.003

Moldovan S, Arenal R, Ersen O (2015) 3D Nanometric Analyses via Electron Tomography: Application to Nanomaterials. In: Deepak FL, Mayoral A, Arenal R (eds) Advanced Transmission Electron Microscopy: Applications to Nanomaterials. Springer International Publishing, Cham, pp 171-205

Mondini S, Ferretti AM, Puglisi A, Ponti A (2012) PEBBLES and PEBBLEJUGGLER: software for accurate, unbiased, and fast measurement and analysis of nanoparticle morphology from transmission electron microscopy (TEM) micrographs. Nanoscale 4:5356-5372. doi: 10.1039/c2nr31276j

Nakafuji A, Murakami Y, Shindo D (2001) Effect of diffraction condition on mean free path determination by EELS. J Electron Microsc (Tokyo) 50:23-28. doi: 10.1093/jmicro/50.1.23

Nowack B, Bucheli TD (2007) Occurrence, behavior and effects of nanoparticles in the environment. Environ Pollut 150:5-22. doi: 10.1016/j.envpol.2007.06.006

Pace HE, Rogers NJ, Jarolimek C, et al (2012) Single Particle Inductively Coupled Plasma-Mass Spectrometry: A Performance Evaluation and Method Comparison in the Determination of Nanoparticle Size. Environ Sci Technol 46:12272-12280. doi: 10.1021/es301787d

Park C, Huang JZ, Ji JX, Ding Y (2013) Segmentation, Inference and Classification of Partially Overlapping Nanoparticles. IEEE Trans Pattern Anal Mach Intell 35:1-1. doi: 10.1109/TPAMI.2012.163

ParticleLab How to prepare a TEM sample by centrifugation. https://www.youtube.com/watch?v=PplB1J7zCCA. Accessed 21 Mar 2019

Plitzko JM, Mayer J (1999) Quantitative thin film analysis by energy filtering transmission electron microscopy. Ultramicroscopy 78:207-219. doi: 10.1016/S0304-3991(99)00021-2

Potapov PL (2014) The experimental electron mean-free-path in Si under typical (S)TEM conditions. Ultramicroscopy 147:21-24. doi: 10.1016/j.ultramic.2014.05.010

Potenza MAC, Krpetić Ž, Sanvito T, et al (2017) Detecting the shape of anisotropic gold nanoparticles in dispersion with single particle extinction and scattering. Nanoscale 9:2778-2784. doi: 10.1039/C6NR08977A

Prasad A, Lead JR, Baalousha M (2015) An electron microscopy based method for the detection and quantification of nanomaterial number concentration in environmentally relevant media. Sci Total Environ 537:479-486. doi: 10.1016/j.scitotenv.2015.07.117

Pyrz WD, Buttrey DJ (2008) Particle Size Determination Using TEM: A Discussion of Image Acquisition and Analysis for the Novice Microscopist. Langmuir 24:11350-11360. doi: 10.1021/la801367j

Reimer L (ed) (1995) Energy-Filtering Transmission Electron Microscopy. Springer-Verlag, Berlin Heidelberg

Roco MC, Bainbridge WS (2005) Societal implications of nanoscience and nanotechnology: Maximizing human benefit. J Nanoparticle Res 7:1-13. doi: 10.1007/s11051-004-2336-5

Savitzky A, Golay M (1964) Smoothing + Differentiation of Data by Simplified Least Squares Procedures. Anal Chem 36:1627-. doi: 10.1021/ac60214a047

Schindelin J, Arganda-Carreras I, Frise E, et al (2012) Fiji: an open-source platform for biological-image analysis. Nat Methods 9:676-682. doi: 10.1038/nmeth.2019 
Schneider CA, Rasband WS, Eliceiri KW (2012) NIH Image to ImageJ: 25 years of image analysis. Nat Methods 9:671-675. doi: 10.1038/nmeth.2089

Schöpe HJ, Marnette O, van Megen W, Bryant G (2007) Preparation and Characterization of Particles with Small Differences in Polydispersity. Langmuir 23:11534-11539. doi: 10.1021/la7018132

Tan YZ, Cheng A, Potter CS, Carragher B (2016) Automated data collection in single particle electron microscopy. Microsc Oxf Engl 65:43-56. doi: 10.1093/jmicro/dfv369

Thomas JM, Midgley PA, Ducati C, Leary RK (2013) Nanoscale electron tomography and atomic scale highresolution electron microscopy of nanoparticles and nanoclusters: A short surveyNanoscale electron tomography and atomic scale high-resolution electron microscopy of nanoparticles and nanoclusters: A short surveyretain-->. Prog Nat Sci Mater Int 23:222-234. doi: 10.1016/j.pnsc.2013.04.003

von der Kammer F, Legros S, Hofmann T, et al (2011) Separation and characterization of nanoparticles in complex food and environmental samples by field-flow fractionation. TrAC Trends Anal Chem 30:425-436. doi: 10.1016/j.trac.2010.11.012

Wagner T (2016) ij-particlesizer: ParticleSizer 1.0.1. Zenodo. 10.5281/zenodo.56457. ij-particlesizer: ParticleSizer 1.0.1. Zenodo. 10.5281/zenodo.56457:

Wagner T, Behnel P (2015) ij-non-local-means: ImageJ Plugin for Non-Local-Means Filtering. Figshare. doi: http://dx.doi.org/10.6084/m9.figshare.878038.v6

Wick S, Baeyens B, Fernandes MM, Voegelin A (2018) Thallium Adsorption onto Illite. Environ Sci Technol 52:571-580. doi: 10.1021/acs.est.7b04485

Wiesner MR, Lowry GV, Alvarez P, et al (2006) Assessing the risks of manufactured nanomaterials. Environ Sci Technol 40:4336-4345. doi: 10.1021/es062726m 